Research Article

\title{
Evaluation of the COVID-19 Pandemic Intervention Strategies with Hesitant F-AHP
}

\author{
Funda Samanlioglu $(\mathbb{D}$ ) and Burak Erkan Kaya \\ Department of Industrial Engineering, Kadir Has University, Cibali 34083, Istanbul, Turkey \\ Correspondence should be addressed to Funda Samanlioglu; fsamanlioglu@khas.edu.tr
}

Received 18 May 2020; Revised 28 July 2020; Accepted 4 August 2020; Published 20 August 2020

Academic Editor: Pasi A. Karjalainen

Copyright (C) 2020 Funda Samanlioglu and Burak Erkan Kaya. This is an open access article distributed under the Creative Commons Attribution License, which permits unrestricted use, distribution, and reproduction in any medium, provided the original work is properly cited.

\begin{abstract}
In this study, a hesitant fuzzy AHP method is presented to help decision makers (DMs), especially policymakers, governors, and physicians, evaluate the importance of intervention strategy alternatives applied by various countries for the COVID-19 pandemic. In this research, a hesitant fuzzy multicriteria decision making (MCDM) method, hesitant fuzzy Analytic Hierarchy Process (hesitant F-AHP), is implemented to make pairwise comparison of COVID-19 country-level intervention strategies applied by various countries and determine relative importance scores. An illustrative study is presented where fifteen intervention strategies applied by various countries in the world during the COVID-19 pandemic are evaluated by seven physicians (a professor of infectious diseases and clinical microbiology, an infectious disease physician, a clinical microbiology physician, two internal medicine physicians, an anesthesiology and reanimation physician, and a family physician) in Turkey who act as DMs in the process.
\end{abstract}

\section{Introduction}

As was realized from the previous 2009 AH1N1 pandemic and the recent COVID-19 pandemic, countries need efficient mitigation planning to prevent mass infection and fatalities. An effective intervention plan may help flatten the epidemic curve and, with protective measures, there might be a delay and reduction in the peak of the outbreak. As a result, the number of cases at any time stays under the surge capacity of a country's healthcare system. If the surge capacity of a country's healthcare system is exceeded, the morbidity and mortality rates increase for all hospitalized patients, not just for COVID-19 cases.

The basic reproduction number $\left(R_{0}\right)$ is the key factor that shows the strength of the epidemic; it is, without any interventions, the mean number of secondary cases generated by a single infected case in a population with no immunity to infection [1]. When $R_{0}$ is greater than 1 , the epidemic takes hold, and the overall fraction of population likely to be infected without interventions is the area under the epidemic curve which can be calculated roughly with $1-1 / R_{0}$ [2].
Effective intervention strategies, if followed properly, might reduce the $R_{0}$ below 1 and control the spread of COVID-19.

Countries need a systematic approach to determine which intervention strategies to apply during the COVID-19 pandemic and future potential epidemiological waves and pandemics. In this study, intervention strategies applied by countries during the COVID-19 pandemic are evaluated in terms of importance with the help of an MCDM method, hesitant F-AHP. Scenarios for the potential spread and impact of COVID-19 in the EU/EEA, with suggested actions for containment, were given in Johnson et al.'s article [3]. Also, Cheng et al. [4] presented an extensive dataset of government responses to the COVID-19 pandemic, and the interventions that are evaluated in this study are taken from their research. A list of these interventions is presented in Table 1 with detailed explanations and country examples.

In the literature, there is a limited number of studies that focus on the evaluation of intervention strategies. Aledort et al. [5] evaluated the evidence base for nonpharmaceutical public health interventions in an influenza pandemic with the help of literature review and expert opinions. Ciofi degli 
TABLE 1: COVID-19 intervention strategies and country examples.

A1 Quarantine/lockdown of patients and those suspected of
infection
A2 Internal border restrictions reducing the ability to move
freely (transportation) within a country

A3 Social distancing

A4 Health monitoring

A5 Public awareness campaigns

Restriction of nonessential businesses

A7 Restrictions of mass gatherings

External border restrictions reducing the ability to exit or enter a country

Closure of schools

A10

Government policies that affect the country's health resources (materials and health worker)

Formation of new task units/bureaus and government

A11 policies changing administrative capacity to respond to the crisis

A12

Common health testing (independent of suspected infection)

A13 Curfew

A14 Restriction of nonessential government services
Policies to quarantine or shelter in place for at least 14 days. For example, "Hong Kong, a semiautonomous Chinese region, requires travelers from all countries to self-quarantine for 14 days"

Government policies which reduce the ability to move freely within a country. For example, in Peru as of March 15 2020, "officials are also restricting the movement of people across provinces"

Government policies that limit physical contact between individuals to 1.5 meters or 6 feet. For example, in Germany, "a 1.5 meter (4.9 feet) distance should be kept at all times when in public"

Government policies that seek to monitor the health of individuals who are afflicted with or who are likely to be afflicted with the coronavirus. For example, "Taiwan CDC monitors all individuals who had traveled to Wuhan within 14 days and exhibited a fever or symptoms of upper respiratory tract infections"

Efforts to disseminate and convey reliable information about COVID-19, including ways to prevent or mitigate the health effects of COVID-19. For example, on March 22, 2020, it was announced that "the Provincial Youth Council in Namibia carried out an intense public awareness campaign on methods of disease prevention, during which, young associates distributed pamphlets with statements about the pandemic and ways of prevention"

Government policies that restrict nonessential commercial activity. For example, in Serbia, "as of March 21, 2020, the following measures are in effect: supermarkets, gas stations, restaurants, post offices, banks and other service providers will be reducing their hours to observe the curfew, with some closing at 6:00 PM or earlier. Cafes, restaurants and shopping centers are closed, however food delivery is allowed"

Government policies that limit the number of people allowed to congregate in a place. For example, on March 16, 2020, in the United States, "the latest recommendation announced Monday by the federal government to promote social distancing and limit the transmission of the coronavirus is: no more than 10 people in one place"

Government policies which reduce the ability to access ports of entry to or exit from a country. For example, "Namibian government suspends inbound and outbound flights for 30 days"

Government policy that closes educational establishments in a country. For example, in Slovakia, as of March 12, 2020, "all schools and educational establishments will be shut down"

Government policies which affect the material (e.g., medical equipment, number of hospitals for public health) or human (e.g., doctors, nurses) health resources of a country. For example, "Taiwan bans exports of face masks; ban extended to the end of April (2020)" or "Government approves plan to build 60 production lines to make an additional 6 million masks per day"

Government policy that changes the administrative capacity of a part of government to respond to the crisis. For example, on January 20, 2020, "Taiwan activated the Central Epidemic Command Center (CECC) which mobilizes government funds and military personnel to facilitate face mask production"

Government policies which seek to sample large populations for coronavirus regardless of suspected likelihood of affliction with coronavirus.

Government policies that limit domestic freedom of movement to certain times of the day. For example, in Serbia, "as of March 21, 2020 the following measures are in effect: curfew for all residents with few exceptions from 8:00pm to 5:00am the next day"

Government policy that restricts nonessential government services. For example, in Malaysia, from March 18, 2020, to March 31, 2020, "all government and private services except those involved in essential services such as water, electricity, power, telecommunications, postal, transportation, fuel, finance, banking, health, pharmacy, fire, port, airport, security, retail and food supply will also be closed" The head of government declares a state of national emergency. For example, on March 15, 2020, in South Africa: "President Ramaphosa announces national state of disaster" 
Atti et al. [6] evaluated the diffusion of pandemic influenza in Italy and the impact of various control measures with the help of SEIR (Susceptible-Exposed-Infected-Recovered) and individual-based models. Ajelli et al. [7] presented the realtime modeling analysis to estimate the impact of the pandemic and the mitigation measures during the 2009 A/H1N1v pandemic in Italy. Kohlhoff et al. [8] carried out an observational study and evaluated hospital mass screening and infection control practices with a pandemic influenza full-scale exercise in three acute care hospitals in Brooklyn, NY. Ventresca and Aleman [9] investigated the effects of six vaccination strategies in terms of the ability to contain disease spread by constructing a representative social network from the census of the Greater Toronto Area. Schiavo et al. [10] presented a review about evidence on interventions to communicate risk and promote disease mitigation measures in epidemics. Russell et al. [11] conducted a household survey in a school district of Kentucky to evaluate the effect of school closure mitigation on the transmission of influenza-like illness. Luca et al. [12] developed a stochastic spatial age-specific metapopulational model to investigate the impact of school closure on seasonal influenza epidemics in Belgium. Nicolaides [13] modified the SIR (Susceptible-Infected-Recovered) epidemic model to reflect the effects of hand washing in the infection process and investigated the effect of hand-hygiene mitigation strategy at airports for flu-type viruses.

MCDM methods have been rarely utilized to evaluate interventions. Shin et al. [14] used AHP to decide if private clinics and hospitals or public health centers should offer free vaccination to children in Korea. Mourits et al. [15] implemented EVAMIX (evaluations with mixed data) to rank control strategies for classical swine fever epidemics in the EU. Aenishaenslin et al. [16] implemented D-Sight (PROMETHEE with GAIA) to evaluate prevention and control strategies for the Lyme disease in Quebec, Canada. Pooripussarakul et al. [17] applied best-worst scaling to evaluate vaccines in Thailand. Previously, Samanlioglu [1] evaluated influenza intervention strategies in Turkey with fuzzy AHP-VIKOR.

In this study, various intervention strategies applied by countries in the world during the COVID-19 pandemic are evaluated by seven physicians with different expertise, acting as consultants and decision makers (DMs). For pairwise comparison of importance of strategies, as the MCDM method, hesitant F-AHP is applied. With (hesitant fuzzy) AHP, utilizing pairwise comparisons of alternatives and consistency check of these comparisons, dependable alternative scores can be determined. In this research, hesitant F-AHP is preferred over AHP or fuzzy AHP (F-AHP) since, different from AHP, with F-AHP, the uncertainty and vagueness on DMs' judgments can also be captured. Moreover, with the usage of multiple linguistic expressions and "hesitant" terminologies in hesitant F-AHP, more flexibility is attained in decision making than F-AHP since the degree of hesitation that DMs might have in reality can also be reflected.

\section{Literature Review}

In AHP [18], with its multilevel and hierarchical structure, alternatives are evaluated with respect to each criterion with pairwise comparisons and ranked based on a total weighted score. To reflect the obscurity and fuzziness of DMs' judgments, utilizing the concepts of fuzzy set theory $[19,20]$, F-AHP was developed and is used in many MCDM problems in the literature [21-24]. Fuzzy set theory contains classes with unsharp boundaries $[25,26]$, and crisp theory sets can be fuzzified by implementing the fuzzy set concepts [19]. In the literature, different extensions of fuzzy sets, such as intuitionistic fuzzy sets $[27,28]$, Pythagorean fuzzy sets [29-31], picture fuzzy sets [32-35], spherical fuzzy sets [36-43], and hesitant fuzzy sets [44-47], were used to deal with uncertainties in decision making problems.

In F-AHP, for pairwise comparisons, DMs give a single linguistic expression; and this does not reflect the hesitations DMs might have in reality. However, in hesitant F-AHP, DM might utilize hesitant fuzzy set (HFS) concepts [44, 45], hesitant fuzzy linguistic term sets (HFLTS) $[44,46]$, and multiple linguistic expressions and "hesitant" terminologies in their evaluations, which increase the flexibility and accuracy of the decision making process [47]. For example, while comparing interventions 1 and 8 pairwise, DM might give the following assessment: "intervention 1 is between absolutely strong and very strong in comparison to criterion 8".

Hesitant F-AHP was implemented in several MCDM problems in the literature. Some of these applications are assessment of suppliers [48], cargo company performance [49], woodwork manufacturing CNC routers [50], sustainability of hydrogen production methods [51], summer sport schools [52], power generation enterprises [47], and innovation projects [53].

Until now, to the best of the authors' knowledge, hesitant F-AHP has never been studied for the evaluation of intervention strategies. With the proposed hesitant F-AHP, importance of countries' COVID-19 intervention strategies is systematically evaluated. Application steps of hesitant F-AHP are explained in Section 3. An illustrative study is given in Section 4 to demonstrate the implementation, along with the conclusion and discussion in Section 5.

\section{Proposed Hesitant F-AHP Approach}

In the proposed hesitant F-AHP, triangular fuzzy numbers (TFNs) are implemented due to their uncomplicatedness. A fuzzy number is a special fuzzy set $F=\left\{\left(x, \mu_{F}(x)\right), x \in R\right\}$, where $R:-\infty<x<+\infty$ and $\mu_{F}(x)$ is from $R$ to $[0,1]$. A TFN, $\widetilde{M}=(l, m, u) l \leq m \leq u$, has the triangular type membership function 


$$
\mu_{F}(x)= \begin{cases}0, & x<l, \\ \frac{x-l}{m-l}, & l \leq x \leq m, \\ \frac{u-x}{u-m}, & m \leq x \leq u, \\ 0, & x>u .\end{cases}
$$

Arithmetic operations between two positive TFNs $\widetilde{C}=\left(l_{1}, m_{1}, u_{1}\right), \widetilde{D}=\left(l_{2}, m_{2}, u_{2}\right) l_{1} \leq m_{1} \leq u_{1} l_{2} \leq m_{2} \leq u_{2}$ and a crisp number $E$ are explained as [53-55]

$$
\begin{aligned}
\widetilde{C}+\widetilde{D} & =\left(l_{1}+l_{2}, m_{1}+m_{2}, u_{1}+u_{2}\right), \\
\widetilde{C}+E & =\left(l_{1}+E, m_{1}+E, u_{1}+E\right), \\
\widetilde{C}-\widetilde{D} & =\left(l_{1}-u_{2}, m_{1}-m_{2}, u_{1}-l_{2}\right), \\
\widetilde{C}-E & =\left(l_{1}-E, m_{1}-E, u_{1}-E\right), \\
\widetilde{C} * \widetilde{D} & =\left(l_{1} * l_{2}, m_{1} * m_{2}, u_{1} * u_{2}\right), \\
\widetilde{C} * E & =\left(l_{1} * E, m_{1} * E, u_{1} * E\right), \quad \text { for } E \geq 0, \\
\frac{\widetilde{C}}{\widetilde{D}} & =\left(\frac{l_{1}}{u_{2}}, \frac{m_{1}}{m_{2}}, \frac{u_{1}}{l_{2}}\right), \\
\widetilde{C} & =\left(\min \left(\frac{l_{1}}{l_{2}}, \frac{l_{1}}{u_{2}}, \frac{u_{1}}{l_{2}}, \frac{u_{1}}{u_{2}}\right), \frac{m_{1}}{m_{2}}, \max \left(\frac{l_{1}}{l_{2}}, \frac{l_{1}}{u_{2}}, \frac{u_{1}}{l_{2}}, \frac{u_{1}}{u_{2}}\right)\right),
\end{aligned}
$$

if $\widetilde{C}$ and $\widetilde{D}$ are two TFNs (not necessarily positive TFNs).

$$
\begin{aligned}
\frac{\widetilde{C}}{E} & =\left(\frac{l_{1}}{E}, \frac{m_{1}}{E}, \frac{u_{1}}{E}\right), \quad \text { for } E>0, \\
\widetilde{C}^{-1} & =\left(\frac{1}{u_{1}}, \frac{1}{m_{1}}, \frac{1}{l_{1}}\right),
\end{aligned}
$$

$$
\begin{aligned}
\operatorname{MAX}(\widetilde{C}+\widetilde{D}) & =\left(\max \left(l_{1}, l_{2}\right), \max \left(m_{1}, m_{2}\right), \max \left(u_{1}, u_{2}\right)\right), \\
\operatorname{MIN}(\widetilde{C}+\widetilde{D}) & =\left(\min \left(l_{1}, l_{2}\right), \min \left(m_{1}, m_{2}\right), \min \left(u_{1}, u_{2}\right)\right) .
\end{aligned}
$$

For defuzzification of TFNs, "the graded mean integration approach" [56] is applied as

$$
\operatorname{Crisp}(\widetilde{C})=\frac{\left(4 m_{1}+l_{1}+u_{1}\right)}{6} \text {. }
$$

In Triangular Fuzzy Hesitant Fuzzy Sets (TFHFS), the membership degree of an element to a given set is expressed by several possible TFNs. Several aggregation operators for TFHFS were introduced by $\mathrm{Yu}$ [57] for assessment of teaching quality. by

If $X$ is a fixed set, the HFS on $X$ returns a subset of $[0,1]$

$$
G=\left\{<x, h_{G}(x)>\mid x \in X\right\},
$$

where $h_{G}(x)$ is the possible membership degrees of element $x \in X$ to set $G$ with values in $[0,1]$. The lower and upper bounds are calculated as

$$
\begin{aligned}
& h_{(x)}^{-}=\min h(x), \\
& h_{(x)}^{+}=\max h(x) .
\end{aligned}
$$

Basic operations for $3 \mathrm{HFS}, h, h 1, h 2$, are given as

$$
\begin{aligned}
h^{\ddot{u}} & =\bigcup_{\gamma \in h}\left\{\gamma^{\ddot{u}}\right\}, \\
\ddot{u} h & =\bigcup_{\gamma \in h}\left\{1-(1-\gamma)^{\ddot{u}}\right\}, \\
h_{1} \pm h_{2} & =\bigcup_{\gamma 1 \in h 1, \gamma 2 \in h 2}\left\{\gamma^{1}+\gamma^{2}-\gamma^{1} \gamma^{2}\right\}, \\
h_{1} \cap h_{2} & =\bigcup_{\gamma 1 \in h 1, \gamma 2 \in h 2} \min \left\{\gamma^{1}, \gamma^{2}\right\}, \\
h_{1} \bigcup h_{2} & =\bigcup_{\gamma 1 \in h 1, \gamma 2 \in h 2} \max \left\{\gamma^{1}, \gamma^{2}\right\}, \\
h_{1} \otimes h_{2} & =\bigcup_{\gamma 1 \in h 1, \gamma 2 \in h 2}\left\{\gamma^{1} \gamma^{2}\right\} .
\end{aligned}
$$

"Ordered Weighting Averaging (OWA)" operator that can be employed is

$$
\operatorname{OWA}\left(a_{1}, a_{2}, \ldots, a_{n}\right)=\sum_{j=1}^{n} w_{j} b_{j},
$$

where $b_{j}$ is the $j$ th largest of $a_{1}, a_{2}, \ldots, a_{n}$, $w_{j} \in[0,1] \forall j$, and $\sum_{j=1}^{n} w_{j}=1[53,58]$.

3.1. Fuzzy Envelope Approach in Hesitant F-AHP. "Fuzzy envelope approach" [59] is applied to combine DM evaluations in hesitant F-AHP. Scales given for DM evaluations are sorted from the lowest $s_{o}$ to the highest $s_{g}$, so if the DM's evaluations are between $s_{i}$ and $s_{j}$, then $s_{o} \leq s_{i} \leq s_{j} \leq s_{g}$.

Based on the HFLTS, linguistic expressions can be represented by a triangular fuzzy membership function $\widetilde{A}=$ $(a, b, c)$, where $a, b$, and $c$ are calculated as

$$
a=\min \left\{a_{L}^{i}, a_{M}^{i}, a_{M}^{i+1}, \cdots, a_{M}^{j}, a_{R}^{j}\right\}=a_{L}^{i},
$$

$$
\begin{aligned}
& b=\left\{\begin{array}{l}
a_{M}^{i} i f i+1=j, \\
\text { OWA }_{W}\left\{a_{M}^{i}, a_{M}^{i+1}, \cdots, a_{M}^{j}\right\}, \quad \text { otherwise, }
\end{array}\right. \\
& c=\max \left\{a_{L}^{i}, a_{M}^{i}, a_{M}^{i+1}, \cdots, a_{M}^{j}, a_{R}^{j}\right\}=a_{R}^{j} .
\end{aligned}
$$

Weight vector in OWA operator [60] is defined as

$$
w_{1}=\alpha^{n-1}, w_{2}=(1-\alpha) \alpha^{n-2}, \cdots, w_{n}=(1-\alpha),
$$

where $\alpha=(l-j+i) /(l-1)$.

Here, $l$ depends on the number of terms in DM's evaluation scale (in Table 2), $j$ is the rank of the highest, and $i$ is the rank of the lowest evaluation value. $i$ and $j$ can take ranks starting from 0 to $l$ and $n=j-i[53,58]$. 
TABLE 2: Scale for the evaluation of importance of intervention strategy alternatives in hesitant F-AHP [53].

\begin{tabular}{lc}
\hline Linguistic terms & Triangular fuzzy number (TFN) \\
\hline Absolutely strong (AS) & $(2,5 / 2,3)$ \\
Very strong (VS) & $(3 / 2,2,5 / 2)$ \\
Fairly strong (FS) & $(1,3 / 2,2)$ \\
Slightly strong (SS) & $(1,1,3 / 2)$ \\
Equal (E) & $(1,1,1)$ \\
Slightly weak (SW) & $(2 / 3,1,1)$ \\
Fairly weak (FW) & $(1 / 2,2 / 3,1)$ \\
Very weak (VW) & $(2 / 5,1 / 2,2 / 3)$ \\
Absolutely weak (AW) & $(1 / 3,2 / 5,1 / 2)$ \\
\hline
\end{tabular}

In the proposed hesitant F-AHP approach, the DMs make pairwise comparisons of importance of intervention strategy alternatives using the linguistic terms given in Table 2.

Steps of the proposed hesitant F-AHP are as follows:

Step 1. Identify $K$ DMs, $n$ alternatives (intervention strategies), and linguistic terms and scale for the pairwise comparison of alternatives. Each DM makes pairwise comparison of alternatives (intervention strategies) with respect to the importance criterion. Based on the scale used in Table 2 and utilizing equations (8)-(12), DM's assessments are combined with fuzzy envelope approach, and TFNs corresponding to the assessment of each DM are obtained. Calculate

$$
\tilde{x}_{i j}=\frac{1}{K}\left(\tilde{x}_{i j}^{1}(+) \tilde{x}_{i j}^{2}(+) \cdots(+) \tilde{x}_{i j}^{K}\right),
$$

where $\tilde{x}_{i j}^{K}=\left(a_{i j}^{K}, b_{i j}^{K}, c_{i j}^{K}\right) \forall i, j, k$ is the TFN corresponding to the evaluation of the Kth DM.

Step 2.

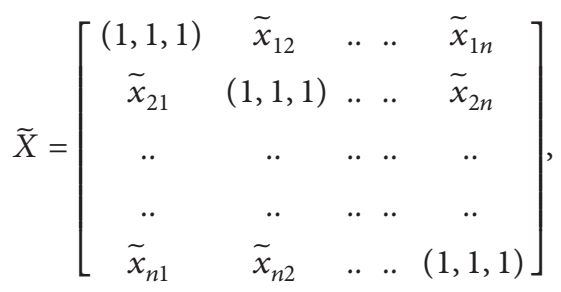

with elements $\tilde{x}_{i j}=\left(a_{i j}, b_{i j}, c_{i j}\right)$ being then defuzzified with equation (4) and approximate alternative scores $w=\left(w_{1}, w_{2}, \ldots, w_{n}\right)$ being determined by averaging the entries on each row of normalized $X$. Therefore, the normalized principal eigenvector is $w$. The largest eigenvalue (principal eigenvalue, $\lambda_{\max }$ ) is determined with

$$
X w^{T}=\lambda_{\max } w^{T} .
$$

The consistency index $(C I)$ is

$$
C I=\frac{\lambda_{\max }-n}{n-1} .
$$

The consistency ratio $(C R)$ is then used to assess the consistency of pairwise comparisons:

$$
C R=\frac{C I}{R I}
$$

$R I$ is the random index, and if $C R<0.10$, the comparisons are consistent and acceptable; otherwise, they are not [18].

Step 3. If comparisons are acceptable, rank the alternatives (intervention strategies) from the best to the worst based on approximate alternative scores $w=\left(w_{1}, w_{2}, \cdots, w_{n}\right)$ in decreasing order. Note that higher $w$ shows better alternative.

\section{Illustrative Study}

In this study, 15 intervention strategies (A1, $\cdots, A 15)$ applied by countries worldwide during the COVID-19 pandemic are evaluated and compared in terms of the importance criterion by 7 physicians who act as DMs. In this study, DMs are a professor of infectious diseases and clinical microbiology (DM1), an infectious disease physician (DM2), a clinical microbiology physician (DM3), two internal medicine physicians (DM4 and DM5), a family physician (DM6), and an anesthesiology and reanimation physician (DM7) in Turkey.

In the proposed hesitant F-AHP, 7 DMs compare intervention strategy alternatives pairwise with the help of linguistic terms in Table 2, and the comparison is given in Table 3. After the combination of each DM's assessments with "fuzzy envelope approach" and aggregation of the corresponding TFNs of $7 \mathrm{DMs}$ assessments, the fuzzy evaluation matrix for the alternative scores $(\widetilde{X})$ in Table 4 is obtained.

Then, elements of $X$ are defuzzified with equation (4), and evaluation matrix $X$ in Table 5 is obtained. Afterwards, $w=\left(w_{1}, w_{2}, \ldots, w_{n}\right)$ is determined by taking the average of the entries on each row of normalized $X . \lambda_{\max }=$ and CR of $X$ is checked with equations (15)-(17) as $C I=(16.268-15) /$ $14=0.0906$ and $C R=C I / R I=0.0906 / 1.59=0.05698$. Since $C R<0.1$, the pairwise comparisons are consistent and acceptable.

Based on the $w=\left(w_{1}, w_{2}, \ldots, w_{n}\right)$ obtained with hesitant F-AHP, intervention strategy alternatives are ranked in terms of importance criterion from the best to the worst as follows: declaration of emergency (A15), quarantine/lockdown of patients and those suspected of infection (A1), curfew (A13), common health testing (independent of suspected infection) (A12), social distancing (A3), closure of schools (A9), external border restrictions reducing the ability to exit or enter a country (A8), internal border restrictions reducing the ability to move freely (transportation) within a country (A2), restrictions of mass gatherings (A7), health monitoring (A4), restriction of nonessential government services (A14), government policies that affect the country's health resources (materials and health worker) (A10), formation of new task units/bureaus 
TABLE 3: Pairwise comparison of importance of COVID-19 intervention strategies by 7 DMs.

\begin{tabular}{|c|c|c|c|c|c|c|c|c|c|c|c|c|c|c|c|}
\hline & $\mathrm{A} 1$ & A2 & $\mathrm{A} 3$ & A4 & A5 & A6 & A7 & A8 & A9 & A10 & A11 & A12 & A13 & A14 & A15 \\
\hline \multirow{7}{*}{ A1 } & $\mathrm{E}$ & FS & FW & FS & FS & VS & $\mathrm{E}$ & FS & AW & $\mathrm{E}$ & $\mathrm{E}$ & $\mathrm{AW}$ & AW & FS & FW \\
\hline & E & VS & VS & AS-VS & $\begin{array}{l}\text { VS- } \\
\text { FS }\end{array}$ & VS & SS & SS & $\mathrm{E}$ & E & SS & $\begin{array}{l}\text { SW- } \\
\text { FW }\end{array}$ & FW-VW & FW & VW \\
\hline & $\mathrm{E}$ & AS & AS & AS & AS & AS & AS & AS & AS & AS & AS & AS & AS & AS & AS \\
\hline & $\mathrm{E}$ & AS & FS & AS & FS-SS & FS & AS & AS & AS & AS-FS & FS & AS & FS-SS & FS & FS-SS \\
\hline & $\mathrm{E}$ & VS & AS & AS & AS & VS & VS & SS & VS & SS & $\mathrm{E}$ & SS & FW & $\mathrm{E}$ & AW \\
\hline & $\mathrm{E}$ & AS & AS & AS & AS & AS & AS & AS-VS & SS & $\mathrm{E}$ & SS & $\mathrm{E}$ & $\mathrm{E}$ & FS & $\mathrm{E}$ \\
\hline & $\mathrm{E}$ & FS-SS & FW & FS & VS & AS & SS-E & $\mathrm{E}$ & FW & SW-FW & VS & FS & VS-FS & VS & AW \\
\hline \multirow{7}{*}{$\mathrm{A} 2$} & & $\mathrm{E}$ & SW & FS & FS & SW & SW & $E$ & $\mathrm{AW}$ & SW & FW & $\mathrm{AW}$ & AW & FW & FW \\
\hline & & $\mathrm{E}$ & SW-E & $\mathrm{E}$ & SW & SW & FW & FW & VW & $\mathrm{E}$ & SW & VW & VW & VW & AW \\
\hline & & $\mathrm{E}$ & VS & VS & AS & $\mathrm{E}$ & $\mathrm{E}$ & $\mathrm{E}$ & E-SW & FW & SW-FW & VW & E-SW & E-SW & FW \\
\hline & & $\mathrm{E}$ & FS & SS & SS-VS & AS & FS & AS & AS & AS-FS & FS & AS & FS-SS & FS & FS \\
\hline & & $\mathrm{E}$ & SW & SW & SS & SW & SW & FW & SW & E & SS & SS & FW & SW & VW \\
\hline & & $\mathrm{E}$ & SW & FW & FW & FS & E-SW & $\mathrm{E}$ & $\mathrm{E}$ & $\mathrm{E}$ & SS & $\mathrm{E}$ & $\mathrm{E}$ & $\mathrm{E}$ & FW \\
\hline & & $\mathrm{E}$ & VS & FS & AS & VS-FS & SS & E-SW & FS & VS & FS & VS-FS & SS-E & FS & SS-E \\
\hline \multirow{7}{*}{ A3 } & & & $\mathrm{E}$ & VS & AS & VS & AS & FS & $\mathrm{E}$ & FS & FS & $\mathrm{E}$ & $\mathrm{E}$ & FS & FS \\
\hline & & & $\mathrm{E}$ & VS & $\begin{array}{l}\text { VS- } \\
\text { FS }\end{array}$ & FS & SS & SS & FW & SW & SW & AW & AW & SW & AW \\
\hline & & & $\mathrm{E}$ & SW-FW & FS & E-SW & SW & $\begin{array}{l}\text { SW- } \\
\text { FW }\end{array}$ & SW-FW & FW & SW & FW & FW & FW & FW-VW \\
\hline & & & E & FS-AS & SS-FS & AS & FS & FS-SS & SS & SS & SS & FW & AS & FS & FS \\
\hline & & & $\mathrm{E}$ & SS & VS & $\mathrm{E}$ & $\mathrm{E}$ & SW & $\mathrm{E}$ & SS & SS & AS & SW & $\mathrm{E}$ & SW \\
\hline & & & $\mathrm{E}$ & $\mathrm{E}$ & VS & FS & $\mathrm{E}$ & $\mathrm{E}$ & $\mathrm{E}$ & SS & FS & $\mathrm{E}$ & FW & FS & SW-FW \\
\hline & & & $\mathrm{E}$ & VS & VS & VS & SS-E & FW & E-SW & VS & AS & FS & FS-SS & VS & SS-E \\
\hline \multirow{7}{*}{ A4 } & & & & $E$ & $E$ & SW & SW & FW & VW & FW & SW & VW & AW & SW & $\mathrm{AW}$ \\
\hline & & & & $\mathrm{E}$ & SS & SW & SW-E & FW & SW & SW & FW & $\mathrm{AW}$ & AW & VW & $\mathrm{AW}$ \\
\hline & & & & $\mathrm{E}$ & AS & AS & SS & SS-E & FS & $\mathrm{E}$ & $\mathrm{E}$ & $\mathrm{E}$ & SS-E & SS-E & $\mathrm{E}$ \\
\hline & & & & $\mathrm{E}$ & FS & FS & FS & FS-SS & FS & FS & SS & FS & SW & SW & SW \\
\hline & & & & $\mathrm{E}$ & SS & SW & SW & FW & SW & SS & $\mathrm{E}$ & VS & SW & SW & VW \\
\hline & & & & $\mathrm{E}$ & AS & AS & VS & $\mathrm{E}$ & $\mathrm{E}$ & SS & FS-SS & $\mathrm{E}$ & $\mathrm{E}$ & FS & $\mathrm{E}$ \\
\hline & & & & $\mathrm{E}$ & SS-E & SS-E & $\begin{array}{l}\text { FW- } \\
\text { VW }\end{array}$ & $\begin{array}{l}\text { FW- } \\
\text { AW }\end{array}$ & VW & SS & FS & SS & SW-FW & FS & AW \\
\hline \multirow{7}{*}{ A5 } & & & & & $\mathrm{E}$ & $\mathrm{E}$ & FW & FW & AW & AW & VW & $\mathrm{AW}$ & AW & SW & AW \\
\hline & & & & & $\mathrm{E}$ & $\mathrm{E}$ & SW & SW & FW & FW & FW & AW & AW & VW & AW \\
\hline & & & & & E & SW & FW & SW & SW & SW-FW & SW & $\begin{array}{l}\text { FW- } \\
\text { VW }\end{array}$ & FW-VW & FW & FW-VW \\
\hline & & & & & E & FS & SS & FS & FS & SS & SS & SW & SS & FS & SS \\
\hline & & & & & $\mathrm{E}$ & SW & SW & AW & SW & SS & SS & FS & SW & SW & AW \\
\hline & & & & & $\mathrm{E}$ & FW & FW & FW & VW & FW & $\mathrm{E}$ & VW & AW & FW & VW \\
\hline & & & & & E & E-SW & VW & AW & VW & SS-E & FS & VS & SS & SW & AW \\
\hline \multirow{7}{*}{ A6 } & & & & & & $\mathrm{E}$ & $E$ & $\mathrm{E}$ & $\mathrm{AW}$ & FW & FW & $\mathrm{AW}$ & AW & $\mathrm{E}$ & AW \\
\hline & & & & & & E & SW-E & $\mathrm{E}$ & FW & SW-E & VW & $\mathrm{AW}$ & AW & FW & AW \\
\hline & & & & & & $\mathrm{E}$ & E-SW & FS & SS & $\mathrm{E}$ & $\mathrm{E}$ & $\mathrm{E}$ & FW-VW & $\mathrm{E}$ & FW-VW \\
\hline & & & & & & E & SW & FW & $\begin{array}{l}\text { AW- } \\
\text { VW }\end{array}$ & SW & SS & VW & FW & E & FW \\
\hline & & & & & & E & SW & AW & SW & SS & SS & FS & SW & SW & AW \\
\hline & & & & & & $\mathrm{E}$ & FW & VW & FW & FW & SW & FW & VW & $\mathrm{E}$ & FW-VW \\
\hline & & & & & & E & $\begin{array}{l}\text { SW- } \\
\text { FW }\end{array}$ & VW & FW & E-SW & $\mathrm{E}$ & VW & VW & SW & AW \\
\hline \multirow{7}{*}{ A7 } & & & & & & & $\mathrm{E}$ & FS & SW & FS & $E$ & VW & AW & FS & AW \\
\hline & & & & & & & $\mathrm{E}$ & SW-E & $\mathrm{E}$ & SW & FW & VW & AW & SW & AW \\
\hline & & & & & & & $\mathrm{E}$ & VS & FS-SS & $\mathrm{E}$ & $\mathrm{E}$ & $\mathrm{E}$ & FW & $\mathrm{E}$ & VW \\
\hline & & & & & & & $\mathrm{E}$ & FW & E & SW & SS & SW & FW & E & FW \\
\hline & & & & & & & $\mathrm{E}$ & SW & $\mathrm{E}$ & SS & SW & FS & FW & E & VW \\
\hline & & & & & & & $\mathrm{E}$ & $\mathrm{E}$ & $\mathrm{E}$ & SW & SS & SW & SW & E & FW \\
\hline & & & & & & & $\mathrm{E}$ & E-SW & $\mathrm{E}$ & VS & AS-VS & VS & VS & VS & SS-E \\
\hline
\end{tabular}


TABle 3: Continued.

\begin{tabular}{|c|c|c|c|c|c|c|c|c|c|c|c|c|c|c|}
\hline $\mathrm{A} 1$ & $\mathrm{~A} 2$ & A3 & A4 & A5 & A6 & A7 & A8 & A9 & A10 & A11 & A12 & A13 & A14 & A15 \\
\hline \multirow{7}{*}{ A8 } & & & & & & & $E$ & FW & SW & FW & AW & AW & VW & $\mathrm{AW}$ \\
\hline & & & & & & & $\mathrm{E}$ & $\mathrm{E}$ & SS & SS & VW & VW & $\mathrm{E}$ & VW \\
\hline & & & & & & & $\mathrm{E}$ & $\mathrm{E}$ & SW & SW & FW & FW-VW & SW & VW \\
\hline & & & & & & & $\mathrm{E}$ & SS & FS & SS & SW & E & $\mathrm{E}$ & $\mathrm{E}$ \\
\hline & & & & & & & $\mathrm{E}$ & AS & FS & FS & AS & VS & AS & SS \\
\hline & & & & & & & $\mathrm{E}$ & $\mathrm{E}$ & SS & SS-FS & FW & SW & $\mathrm{E}$ & SW \\
\hline & & & & & & & $\mathrm{E}$ & AS-VS & VS & AS & VS & FS & $\begin{array}{l}\text { AS- } \\
\text { VS }\end{array}$ & SS-E \\
\hline \multirow{7}{*}{ A9 } & & & & & & & & $\mathrm{E}$ & VS & VS & SW & AW & VS & $\mathrm{AW}$ \\
\hline & & & & & & & & $\mathrm{E}$ & VS & VS & VW & SW & $\mathrm{E}$ & FW \\
\hline & & & & & & & & E & FW-SW & FW-SW & VW & VW & SW-E & VW \\
\hline & & & & & & & & $\mathrm{E}$ & FS & FS & $\mathrm{E}$ & SS & FS & $\mathrm{E}$ \\
\hline & & & & & & & & $\mathrm{E}$ & SS & SW & FS & SW & $\mathrm{E}$ & VW \\
\hline & & & & & & & & $\mathrm{E}$ & SW & SS & SW & $\mathrm{E}$ & $\mathrm{E}$ & SW \\
\hline & & & & & & & & $\mathrm{E}$ & VS & AS & FS & FS-SS & FS & SW-FW \\
\hline \multirow{7}{*}{ A 10} & & & & & & & & & $\mathrm{E}$ & $\mathrm{E}$ & $\mathrm{AW}$ & $\mathrm{AW}$ & FW & $\mathrm{AW}$ \\
\hline & & & & & & & & & $\mathrm{E}$ & $\mathrm{E}$ & FW & VW & VW & AW \\
\hline & & & & & & & & & $\mathrm{E}$ & $\mathrm{E}$ & E & $\mathrm{E}$ & SS-E & $\mathrm{E}$ \\
\hline & & & & & & & & & $\mathrm{E}$ & $\mathrm{E}$ & FW & FS & FS & $\mathrm{E}$ \\
\hline & & & & & & & & & $\mathrm{E}$ & SW & SS & SW & SW & SW \\
\hline & & & & & & & & & $\mathrm{E}$ & $\mathrm{E}$ & SW & SW & SS & SW \\
\hline & & & & & & & & & $\mathrm{E}$ & FS & FW & FW-VW & FW & AW \\
\hline \multirow{7}{*}{ A11 } & & & & & & & & & & $\mathrm{E}$ & $\mathrm{AW}$ & AW & FW & AW \\
\hline & & & & & & & & & & $\mathrm{E}$ & FW & FW & VW & AW \\
\hline & & & & & & & & & & $\mathrm{E}$ & E & $\mathrm{E}$ & E & $\mathrm{E}$ \\
\hline & & & & & & & & & & $\mathrm{E}$ & $\mathrm{E}$ & SS & FS & $\mathrm{E}$ \\
\hline & & & & & & & & & & $\mathrm{E}$ & SS & SW & SW & FW \\
\hline & & & & & & & & & & $\mathrm{E}$ & FW & SW & $\mathrm{E}$ & FW \\
\hline & & & & & & & & & & $\mathrm{E}$ & VW & FW-VW & FW & AW \\
\hline \multirow{7}{*}{ A12 } & & & & & & & & & & & $\mathrm{E}$ & AS & AS & AS \\
\hline & & & & & & & & & & & $\mathrm{E}$ & VS & VS & E \\
\hline & & & & & & & & & & & $\mathrm{E}$ & $\mathrm{E}$ & SS-E & $\mathrm{E}$ \\
\hline & & & & & & & & & & & $\mathrm{E}$ & $\mathrm{E}$ & AS & AS \\
\hline & & & & & & & & & & & $\mathrm{E}$ & FW & FW & AW \\
\hline & & & & & & & & & & & $\mathrm{E}$ & E & SS & E \\
\hline & & & & & & & & & & & $\mathrm{E}$ & SW-FW & FS-SS & FW \\
\hline \multirow{7}{*}{ A13 } & & & & & & & & & & & & $\mathrm{E}$ & AS & AW \\
\hline & & & & & & & & & & & & $\mathrm{E}$ & FS & FW \\
\hline & & & & & & & & & & & & $\mathrm{E}$ & FS & $\mathrm{E}$ \\
\hline & & & & & & & & & & & & $\mathrm{E}$ & AS & $\mathrm{E}$ \\
\hline & & & & & & & & & & & & $\mathrm{E}$ & VS & SW \\
\hline & & & & & & & & & & & & $\mathrm{E}$ & FS & FW \\
\hline & & & & & & & & & & & & $\mathrm{E}$ & $\begin{array}{l}\text { AS- } \\
\text { VS }\end{array}$ & E-SW \\
\hline \multirow{7}{*}{ A14 } & & & & & & & & & & & & & $\mathrm{E}$ & AW \\
\hline & & & & & & & & & & & & & $\mathrm{E}$ & FW \\
\hline & & & & & & & & & & & & & $\mathrm{E}$ & SW \\
\hline & & & & & & & & & & & & & $\mathrm{E}$ & FW \\
\hline & & & & & & & & & & & & & $\mathrm{E}$ & SW \\
\hline & & & & & & & & & & & & & $\mathrm{E}$ & SW-FW \\
\hline & & & & & & & & & & & & & $\mathrm{E}$ & $\begin{array}{l}\text { VW- } \\
\text { AW }\end{array}$ \\
\hline \multirow{6}{*}{ A 15} & & & & & & & & & & & & & & $\mathrm{E}$ \\
\hline & & & & & & & & & & & & & & $\mathrm{E}$ \\
\hline & & & & & & & & & & & & & & $\mathrm{E}$ \\
\hline & & & & & & & & & & & & & & $\mathrm{E}$ \\
\hline & & & & & & & & & & & & & & $\mathrm{E}$ \\
\hline & & & & & & & & & & & & & & $\mathrm{E}$ \\
\hline
\end{tabular}


TABLE 4: The fuzzy evaluation matrix for the intervention strategy alternatives $(\widetilde{X})$.

\begin{tabular}{|c|c|c|c|c|c|}
\hline & A1 & A2 & A3 & A4 & A5 \\
\hline $\mathrm{A} 1$ & $(1.000,1.000,1.000)$ & $(1.571,2.000,2.571)$ & $(1.357,1.763,2.214)$ & $(1.643,2.143,2.714)$ & $(1.500,1.929,2.571)$ \\
\hline $\mathrm{A} 2$ & $(0.399,0.506,0.691)$ & $(1.000,1.000,1.000)$ & $(0.954,1.357,1.571)$ & $(0.953,1.239,1.571)$ & $(1.167,1.586,2.000)$ \\
\hline A3 & $(0.556,0.767,1.024)$ & $(0.757,0.810,1.191)$ & $(1.000,1.000,1.000)$ & $(1.143,1.586,2.000)$ & $(1.357,1.786,2.429)$ \\
\hline A4 & $(0.379,0.477,0.667)$ & $(0.724,0.906,1.167)$ & $(0.601,0.735,1.001)$ & $(1.000,1.000,1.000)$ & $(1.286,1.500,1.929)$ \\
\hline A5 & $(0.399,0.506,0.739)$ & $(0.604,0.846,1.071)$ & $(0.419,0.534,0.787)$ & $(0.595,0.781,0.857)$ & $(1.000,1.000,1.000)$ \\
\hline A6 & $(0.384,0.481,0.644)$ & $(0.747,0.796,1.143)$ & $(0.590,0.677,0.906)$ & $(0.690,0.781,1.071)$ & $(0.929,1.024,1.357)$ \\
\hline A7 & $(0.532,0.671,0.739)$ & $(0.881,1.024,1.357)$ & $(0.738,0.867,1.000)$ & $(0.796,0.953,1.381)$ & $(1.024,1.357,1.786)$ \\
\hline A8 & $(0.548,0.696,0.810)$ & $(0.904,1.057,1.286)$ & $(0.810,0.977,1.357)$ & $(0.881,1.371,1.714)$ & $(1.214,1.524,2.000)$ \\
\hline A9 & $(0.819,1.043,1.239)$ & $(1.047,1.224,1.571)$ & $(0.953,1.071,1.357)$ & $(1.000,1.191,1.571)$ & $(1.214,1.524,2.000)$ \\
\hline A 10 & $(0.762,0.863,1.071)$ & $(0.819,0.949,1.167)$ & $(0.701,0.953,1.167)$ & $(0.787,1.024,1.214)$ & $(1.001,1.357,1.714)$ \\
\hline A11 & $(0.653,0.796,0.881)$ & $(0.763,0.977,1.357)$ & $(0.667,0.820,1.071)$ & $(0.810,0.977,1.214)$ & $(0.906,1.167,1.429)$ \\
\hline A 12 & $(0.833,0.996,1.286)$ & $(1.057,1.343,1.643)$ & $(0.976,1.224,1.500)$ & $(1.010,1.239,1.453)$ & $(1.200,1.524,2.024)$ \\
\hline A13 & $(0.890,1.153,1.571)$ & $(1.095,1.381,1.714)$ & $(0.976,1.224,1.571)$ & $(1.238,1.429,1.857)$ & $(1.334,1.714,2.143)$ \\
\hline A14 & $(0.604,0.773,1.024)$ & $(0.929,1.120,1.500)$ & $(0.700,0.859,1.167)$ & $(0.881,1.049,1.429)$ & $(1.000,1.239,1.714)$ \\
\hline \multirow[t]{2}{*}{ A15 } & $(1.190,1.510,1.857)$ & $(1.095,1.524,1.929)$ & $(0.952,1.191,1.714)$ & $(1.500,1.786,2.143)$ & $(1.596,2.071,2.571)$ \\
\hline & A6 & A7 & A8 & A9 & A10 \\
\hline A1 & $(1.643,2.143,2.643)$ & $(1.500,1.786,2.214)$ & $(1.357,1.643,2.143)$ & $(1.190,1.439,1.786)$ & $(1.071,1.371,1.643)$ \\
\hline $\mathrm{A} 2$ & $(1.001,1.357,1.643)$ & $(0.787,1.024,1.214)$ & $(0.952,1.120,1.286)$ & $(0.867,1.129,1.310)$ & $(0.953,1.300,1.500)$ \\
\hline A3 & $(1.238,1.643,2.000)$ & $(1.096,1.286,1.571)$ & $(0.810,0.977,1.357)$ & $(0.810,0.906,1.071)$ & $(0.953,1.167,1.571)$ \\
\hline $\mathrm{A} 4$ & $(1.144,1.500,1.786)$ & $(0.844,1.143,1.429)$ & $(0.691,0.808,1.214)$ & $(0.734,1.000,1.191)$ & $(0.881,1.024,1.357)$ \\
\hline A5 & $(0.787,1.024,1.143)$ & $(0.606,0.787,1.024)$ & $(0.571,0.806,1.000)$ & $(0.567,0.796,0.977)$ & $(0.690,0.773,1.143)$ \\
\hline A6 & $(1.000,1.000,1.000)$ & $(0.668,0.906,1.000)$ & $(0.661,0.796,0.977)$ & $(0.548,0.687,0.952)$ & $(0.715,0.906,1.071)$ \\
\hline A7 & $(1.000,1.071,1.571)$ & $(1.000,1.000,1.000)$ & $(0.858,1.167,1.357)$ & $(0.953,1.000,1.143)$ & $(0.930,1.214,1.429)$ \\
\hline A8 & $(1.214,1.524,1.857)$ & $(0.843,0.953,1.310)$ & $(1.000,1.000,1.000)$ & $(1.143,1.310,1.643)$ & $(0.977,1.286,1.643)$ \\
\hline A9 & $(1.167,1.571,2.071)$ & $(0.929,0.953,1.071)$ & $(0.762,0.900,1.024)$ & $(1.000,1.000,1.000)$ & $(1.096,1.452,1.857)$ \\
\hline A 10 & $(0.953,1.143,1.500)$ & $(0.796,0.881,1.167)$ & $(0.677,0.834,1.096)$ & $(0.624,0.739,1.073)$ & $(1.000,1.000,1.000)$ \\
\hline A11 & $(0.977,1.214,1.429)$ & $(0.811,0.986,1.167)$ & $(0.667,0.891,1.143)$ & $(0.614,0.724,1.049)$ & $(0.929,0.953,1.071)$ \\
\hline A12 & $(1.357,1.739,2.143)$ & $(0.986,1.167,1.524)$ & $(1.033,1.343,1.739)$ & $(1.000,1.191,1.571)$ & $(1.096,1.429,1.786)$ \\
\hline A13 & $(1.429,1.857,2.429)$ & $(1.200,1.571,2.024)$ & $(1.057,1.310,1.739)$ & $(1.096,1.310,1.643)$ & $(1.143,1.381,1.857)$ \\
\hline A14 & $(1.000,1.071,1.286)$ & $(0.843,0.881,1.024)$ & $(0.881,0.971,1.167)$ & $(0.771,0.834,1.024)$ & $(0.905,1.239,1.571)$ \\
\hline \multirow[t]{2}{*}{ A15 } & $(1.571,2.071,2.714)$ & $(1.381,1.857,2.286)$ & $(1.191,1.500,1.786)$ & $(1.286,1.571,2.071)$ & $(1.429,1.643,2.000)$ \\
\hline & A11 & $\mathrm{A} 12$ & A13 & $\mathrm{A} 14$ & A15 \\
\hline A1 & $(1.214,1.429,1.786)$ & $(1.119,1.367,1.714)$ & $(0.890,1.081,1.571)$ & $(1.143,1.524,1.929)$ & $(0.794,0.924,1.239)$ \\
\hline $\mathrm{A} 2$ & $(0.810,1.048,1.429)$ & $(0.876,1.057,1.406)$ & $(0.700,0.796,1.096)$ & $(0.748,1.024,1.239)$ & $(0.604,0.773,1.096)$ \\
\hline A3 & $(1.049,1.357,1.714)$ & $(0.904,1.106,1.357)$ & $(0.857,1.034,1.357)$ & $(0.953,1.310,1.643)$ & $(0.700,0.939,1.286)$ \\
\hline $\mathrm{A} 4$ & $(0.881,1.024,1.357)$ & $(0.890,1.057,1.310)$ & $(0.643,0.781,0.929)$ & $(0.773,1.071,1.310)$ & $(0.580,0.671,0.739)$ \\
\hline A5 & $(0.796,0.953,1.239)$ & $(0.661,0.900,1.167)$ & $(0.580,0.671,0.929)$ & $(0.630,0.906,1.096)$ & $(0.446,0.514,0.739)$ \\
\hline A6 & $(0.796,0.881,1.096)$ & $(0.566,0.710,0.906)$ & $(0.433,0.567,0.763)$ & $(0.834,0.953,1.000)$ & $(0.374,0.467,0.714)$ \\
\hline A7 & $(0.953,1.096,1.429)$ & $(0.806,1.071,1.263)$ & $(0.619,0.830,1.071)$ & $(1.024,1.214,1.357)$ & $(0.494,0.591,0.834)$ \\
\hline A8 & $(1.024,1.239,1.714)$ & $(0.843,1.106,1.381)$ & $(0.757,0.986,1.239)$ & $(1.081,1.286,1.524)$ & $(0.686,0.771,0.977)$ \\
\hline A9 & $(1.167,1.524,1.929)$ & $(0.734,1.000,1.191)$ & $(0.724,0.843,1.096)$ & $(1.024,1.286,1.500)$ & $(0.543,0.677,0.834)$ \\
\hline A 10 & $(0.953,1.071,1.143)$ & $(0.643,0.773,1.000)$ & $(0.639,0.843,1.024)$ & $(0.724,0.906,1.239)$ & $(0.619,0.743,0.786)$ \\
\hline A11 & $(1.000,1.000,1.000)$ & $(0.676,0.749,0.953)$ & $(0.653,0.796,1.000)$ & $(0.724,0.906,1.096)$ & $(0.570,0.649,0.786)$ \\
\hline $\mathrm{A} 12$ & $(1.167,1.500,1.786)$ & $(1.000,1.000,1.000)$ & $(1.071,1.263,1.500)$ & $(1.286,1.524,2.071)$ & $(1.119,1.296,1.500)$ \\
\hline A13 & $(1.096,1.357,1.786)$ & $(0.819,0.914,1.167)$ & $(1.000,1.000,1.000)$ & $(1.429,1.929,2.500)$ & $(0.667,0.820,0.929)$ \\
\hline A14 & $(1.000,1.239,1.571)$ & $(0.557,0.781,0.953)$ & $(0.413,0.530,0.762)$ & $(1.000,1.000,1.000)$ & $(0.501,0.687,0.881)$ \\
\hline A15 & $(1.429,1.786,2.143)$ & $(0.951,1.114,1.286)$ & $(1.143,1.357,1.714)$ & $(1.214,1.500,2.143)$ & $(1.000,1.000,1.000)$ \\
\hline
\end{tabular}

TABLE 5: $X$ and intervention strategy alternative scores $(w)$.

\begin{tabular}{|c|c|c|c|c|c|c|c|c|c|c|c|c|c|c|c|c|}
\hline & A1 & A2 & $\mathrm{A} 3$ & $\mathrm{~A} 4$ & A5 & A6 & A7 & A8 & A9 & A10 & A11 & A12 & A13 & A14 & A15 & $w$ \\
\hline $\mathrm{A} 1$ & 1.000 & 2.024 & 1.770 & 2.155 & 1.964 & 2.143 & 1.810 & 1.679 & 1.455 & 1.367 & 1.452 & 1.384 & 1.131 & 1.528 & 0.955 & 0.0936 \\
\hline $\mathrm{A} 2$ & 0.519 & 1.000 & 1.326 & 1.246 & 1.585 & 1.345 & 1.016 & 1.120 & 1.115 & 1.275 & 1.072 & 1.085 & 0.830 & 1.014 & 0.799 & 0.0644 \\
\hline A3 & 0.775 & 0.865 & 1.000 & 1.581 & 1.821 & 1.635 & 1.302 & 1.013 & 0.917 & 1.199 & 1.365 & 1.114 & 1.059 & 1.306 & 0.957 & 0.0704 \\
\hline A4 & 0.492 & 0.919 & 0.757 & 1.000 & 1.536 & 1.488 & 1.141 & 0.856 & 0.988 & 1.056 & 1.056 & 1.071 & 0.783 & 1.061 & 0.667 & 0.0580 \\
\hline A5 & 0.527 & 0.843 & 0.557 & 0.763 & 1.000 & 1.004 & 0.796 & 0.799 & 0.788 & 0.821 & 0.974 & 0.905 & 0.699 & 0.891 & 0.540 & 0.0471 \\
\hline A6 & 0.492 & 0.845 & 0.701 & 0.814 & 1.064 & 1.000 & 0.882 & 0.804 & 0.708 & 0.902 & 0.903 & 0.719 & 0.577 & 0.941 & 0.493 & 0.0464 \\
\hline A7 & 0.659 & 1.056 & 0.868 & 0.998 & 1.373 & 1.143 & 1.000 & 1.147 & 1.016 & 1.203 & 1.127 & 1.059 & 0.835 & 1.206 & 0.616 & 0.0604 \\
\hline
\end{tabular}


Table 5: Continued.

\begin{tabular}{|c|c|c|c|c|c|c|c|c|c|c|c|c|c|c|c|c|}
\hline & A1 & 42 & 3 & A4 & A5 & A6 & 47 & A8 & A9 & A10 & A11 & A12 & A13 & A14 & A15 & $w$ \\
\hline 48 & 690 & 070 & & 347 & & & & & & & & & & & & \\
\hline 19 & & & & & & & & & & & & & & & & \\
\hline 10 & & & & & & & & & & & & & & & & \\
\hline 11 & 0 & & & & & & & & & & & & & & & \\
\hline 412 & & & & & & & & & & & & & & & & 799 \\
\hline 1 & & & & & & & & & & & & & & & & 0.0811 \\
\hline 14 & 787 & & & & 1278 & & & & & & & & & & & \\
\hline 1 & .515 & .520 & 1.239 & 1.798 & 2.075 & 2.095 & 1.849 & 1.496 & 1.607 & 1.667 & 1.786 & 1.116 & 1.381 & 1.560 & 1.000 & 0.0938 \\
\hline
\end{tabular}

and government policies changing administrative capacity to respond to the crisis (A11), public awareness campaigns (A5), and restriction of nonessential businesses (A6).

\section{Conclusion and Discussion}

In this paper, a hesitant F-AHP approach is presented to help DMs such as policymakers, governors, and physicians evaluate and rank intervention strategy alternatives applied by various countries during the COVID-19 pandemic. At present, there does not appear to be a study in the literature that evaluates countries' COVID-19 intervention strategies. Moreover, in the literature a systematic MCDM approach such as hesitant F-AHP has never been utilized to evaluate and rank COVID-19 intervention strategies. Adoption of hesitant fuzzy linguistic terms in the process captures the fuzziness and hesitations and provides flexibility in decision making.

In the literature, AHP is mainly criticized due to the possible occurrence of rank reversal phenomenon caused by adding or deleting an alternative [61-64]. Adding a new alternative includes new information in the model, and therefore the decision needs to be reevaluated [65]. Unfortunately, the rank reversal problem occurs not only in AHP, but also in many other decision making approaches such as Borda-Kendall method, SAW, TOPSIS, and crossefficiency evaluation method [61]. However, this limitation did not affect our analysis since we did not need to add or delete any new intervention alternatives.

For the illustrative study, expert opinion for the evaluations was needed, so a professor of infectious diseases and clinical microbiology, an infectious disease physician, a clinical microbiology physician, two internal medicine physicians, a family physician, and an anesthesiology and reanimation physician in Turkey acted as DMs. Based on their evaluation, declaration of emergency, quarantine/ lockdown of patients and those suspected of infection, and curfew are determined as the best three intervention strategies among the evaluated ones.

In this research, intervention strategies are evaluated without taking into consideration the interventions' timing. In reality, the timing of the intervention, with respect to the beginning and peak of the epidemic, and duration of the application of the intervention are really significant. Therefore, when making decisions, DMs need to take those into consideration, as well as intervention strategy rankings. Based on these, the proposed hesitant F-AHP approach can be adopted and utilized by policy makers, governors, national public health departments, and physicians for the evaluation of countries' intervention strategies for COVID19 and other future similar epidemics. Also, for future research, various other potentially conflicting quantitative and qualitative criteria can be taken into consideration, and interactions, dependencies, and feedback relationships between them can be investigated with hesitant fuzzy analytic network process (hesitant F-ANP).

\section{Data Availability}

All the data used to support the findings of this study are included within the article.

\section{Conflicts of Interest}

The author declares that there are no conflicts of interest regarding the publication of this article.

\section{Acknowledgments}

The authors would like to thank Prof. Dr. Önder Ergönül, M.D., M.P.H. (infectious diseases), Burak Kömürcü, M.D. (infectious diseases), Leyla Genç, M.D. (clinical microbiology), Murat Görgülü, M.D. (internal medicine), Tuğba Öztürk, M.D. (internal medicine), Alp Özer, M.D. (family physician), and Sezer Yakupoğlu, M.D. (anesthesiology and reanimation) for their collaboration in this research.

\section{References}

[1] F. Samanlioglu, "Evaluation of influenza intervention strategies in Turkey with fuzzy AHP-VIKOR," Journal of Healthcare Engineering, vol. 2019, Article ID 9486070, 9 pages, 2019.

[2] R. M. Anderson, H. Heesterbeek, D. Klinkenberg, and T. D. Hollingsworth, "How will country-based mitigation measures influence the course of the COVID-19 epidemic?" The Lancet, vol. 395, no. 10228, pp. 931-934, 2020.

[3] H. C. Johnson, C. M. Gossner, E. Colzani et al., "Potential scenarios for the progression of a COVID-19 epidemic in the European Union and the European Economic Area, March 2020," Eurosurveillance, vol. 25, no. 9, pp. 1-5, 2020.

[4] C. Cheng, J. Barceló, A. S. Hartnett, and R. Kubinec, CoronaNet: A Dyadic Dataset of Government Responses to the COVID-19 Pandemic, SocArXiv, Ithaca, NY, USA, pp. 1-62, 2020. 
[5] J. E. Aledort, N. Lurie, J. Wasserman, and S. A. Bozzette, "Non-pharmaceutical public health interventions for pandemic influenza: an evaluation of the evidence base," $B M C$ Public Health, vol. 7, no. 1, pp. 1-9, 2007.

[6] M. L. Ciofi degli Atti, S. Merler, C. Rizzo et al., "Mitigation measures for pandemic influenza in Italy: an individual based model considering different scenarios," PLoS One, vol. 3, no. 3, Article ID e1790, 2008.

[7] M. Ajelli, S. Merler, A. Pugliese, and C. Rizzo, "Model predictions and evaluation of possible control strategies for the 2009 A/H1N1v influenza pandemic in Italy," Epidemiology and Infection, vol. 139, no. 1, pp. 68-79, 2011.

[8] S. A. Kohlhoff, B. Crouch, P. M. Roblin et al., "Evaluation of hospital mass screening and infection control practices in a pandemic influenza full-scale exercise," Disaster Medicine and Public Health Preparedness, vol. 6, no. 4, pp. 378-384, 2012.

[9] M. Ventresca and D. Aleman, "Evaluation of strategies to mitigate contagion spread using social network characteristics," Social Networks, vol. 35, no. 1, pp. 75-88, 2013.

[10] R. Schiavo, M. May Leung, and M. Brown, "Communicating risk and promoting disease mitigation measures in epidemics and emerging disease settings," Pathogens and Global Health, vol. 108, no. 2, pp. 76-94, 2014.

[11] E. S. Russell, Y. Zheteyeva, H. Gao et al., "Reactive school closure during increased influenza-like illness (ILI) activity in Western Kentucky, 2013: a field evaluation of effect on ILI incidence and economic and social consequences for families," Open Forum Infectious Diseases, vol. 3, no. 3, 2016.

[12] G. D. Luca, K. V. Kerckhove, P. Coletti et al., "The impact of regular school closure on seasonal influenza epidemics: a data-driven spatial transmission model for Belgium," $B M C$ Infectious Diseases, vol. 18, no. 1, pp. 1-16, 2018.

[13] C. Nicolaides, D. Avraam, L. Cueto-Felgueroso, M. C. González, and R. Juanes, "Hand-hygiene mitigation strategies against global disease spreading through the air transportation network," Risk Analysis, vol. 40, no. 4, pp. 723-740, 2019.

[14] T. Shin, C.-B. Kim, Y.-H. Ahn et al., "The comparative evaluation of expanded national immunization policies in Korea using an analytic hierarchy process," Vaccine, vol. 27, no. 5, pp. 792-802, 2009.

[15] M. C. M. Mourits, M. A. P. M. van Asseldonk, and R. B. M. Huirne, "Multi Criteria Decision Making to evaluate control strategies of contagious animal diseases," Preventive Veterinary Medicine, vol. 96, no. 3-4, pp. 201-210, 2010.

[16] C. Aenishaenslin, V. Hongoh, H. D. Cissé et al., "Multi-criteria decision analysis as an innovative approach to managing zoonoses: results from a study on Lyme disease in Canada," BMC Public Health, vol. 13, no. 1, pp. 1-16, 2013.

[17] S. Pooripussarakul, A. Riewpaiboon, D. Bishai, C. Muangchana, and S. Tantivess, "What criteria do decision makers in Thailand use to set priorities for vaccine introduction?" BMC Public Health, vol. 16, no. 1, pp. 1-7, 2016.

[18] T. L. Saaty, The Analytic Hierarchy Process, McGraw-Hill, New York, NY, USA, 1980.

[19] L. A. Zadeh, "Fuzzy logic, neural networks, and soft computing," Communications of the ACM, vol. 37, no. 3, pp. 77-84, 1994.

[20] H.-J. Zimmermann, "Fuzzy logic for planning and decision making," F. A. Lootsma, Ed., p. 195, Kluwer Academic Publishers, Dordrecht, Netherlands, 1997.

[21] P. Srichetta and W. Thurachon, "Applying fuzzy analytic hierarchy process to evaluate and select product of notebook computers," International Journal of Modeling and Optimization, vol. 2, no. 2, pp. 168-173, 2012.

[22] D. Choudhary and R. Shankar, "An STEEP-fuzzy AHPTOPSIS framework for evaluation and selection of thermal power plant location: a case study from India," Energy, vol. 42, no. 1, pp. 510-521, 2012.

[23] S. Avikal, P. K. Mishra, and R. Jain, "A Fuzzy AHP and PROMETHEE method-based heuristic for disassembly line balancing problems," International Journal of Production Research, vol. 52, no. 5, pp. 1306-1317, 2013.

[24] G. Kabir and R. S. Sumi, "Power substation location selection using fuzzy analytic hierarchy process and PROMETHEE: a case study from Bangladesh," Energy, vol. 72, pp. 717-730, 2014.

[25] F. A. Lootsma, Fuzzy Logic for Planning and Decision Making, Springer, New York, NY, USA, 1997.

[26] G. J. Klir and B. Yuan, Fuzzy Sets and Fuzzy Logic: Theory and Applications, Springer, New York, NY, USA, 1st edition, 1995.

[27] Z. Xu and R. R. Yager, "Some geometric aggregation operators based on intuitionistic fuzzy sets," International Journal of General Systems, vol. 35, no. 4, pp. 417-433, 2006.

[28] K. T. Atanassov, "Intuitionistic fuzzy sets," Fuzzy Sets and Systems, vol. 20, no. 1, pp. 87-96, 1986.

[29] H. Garg, "New logarithmic operational laws and their aggregation operators for Pythagorean fuzzy set and their applications," International Journal of Intelligent Systems, vol. 34, no. 1, pp. 82-106, 2019.

[30] X. Zhang and Z. Xu, "Extension of TOPSIS to multiple criteria decision making with pythagorean fuzzy sets," International Journal of Intelligent Systems, vol. 29, no. 12, pp. 1061-1078, 2014.

[31] R. R. Yager, "Pythagorean fuzzy subsets," in Proceedings of the Joint IFSA World Congress and NAFIPS Annual Meeting (IFSA/NAFIPS), pp. 57-61, Edmonton, Canada, June 2013.

[32] B. C. Cuong and V. Kreinovich, "Picture fuzzy sets-a new concept for computational intelligence problems," in Proceedings of the Third World Congress on Information and Communication Technologies (WICT 2013), pp. 1-6, Hanoi, Vietnam, December 2013.

[33] T. Nucleus, S. Ashraf, T. Mahmood, S. Abdullah, and Q. Khan, "Picture fuzzy linguistic sets and their applications for multi-attribute group decision making problems," The Nucleus, vol. 55, no. 2, pp. 66-73, 2018.

[34] S. Zeng, S. Asharf, M. Arif, and S. Abdullah, "Application of exponential Jensen picture fuzzy divergence measure in multi-criteria group decision making," Mathematics, vol. 7, no. 2, p. 191, 2019.

[35] S. Ashraf, T. Mahmood, S. Abdullah, and Q. Khan, "Different approaches to multi-criteria group decision making problems for picture fuzzy environment," Bulletin of the Brazilian Mathematical Society, New Series, vol. 50, no. 2, pp. 373-397, 2019.

[36] S. Ashraf, S. Abdullah, and T. Mahmood, "GRA method based on spherical linguistic fuzzy Choquet integral environment and its application in multi-attribute decision-making problems," Mathematical Sciences, vol. 12, no. 4, pp. 263-275, 2018.

[37] S. Ashraf, S. Abdullah, T. Mahmood, F. Ghani, and T. Mahmood, "Spherical fuzzy sets and their applications in multi-attribute decision making problems," Journal of Intelligent \& Fuzzy Systems, vol. 36, no. 3, pp. 2829-2844, 2019.

[38] S. Ashraf, S. Abdullah, and T. Mahmood, "Spherical fuzzy Dombi aggregation operators and their application in group decision making problems," Journal of Ambient Intelligence 
and Humanized Computing, vol. 11, no. 7, pp. 2731-2749, 2020.

[39] S. Ashraf, S. Abdullah, and L. Abdullah, "Child development influence environmental factors determined using spherical fuzzy distance measures," Mathematics, vol. 7, no. 8, p. 661, 2019.

[40] S. Ashraf, S. Abdullah, M. Aslam, M. Qiyas, and M. A. Kutbi, "Spherical fuzzy sets and its representation of spherical fuzzy t-norms and t-conorms," Journal of Intelligent \& Fuzzy Systems, vol. 36, no. 6, pp. 6089-6102, 2019.

[41] H. Jin, S. Ashraf, S. Abdullah, M. Qiyas, M. Bano, and S. Zeng, "Linguistic spherical fuzzy aggregation operators and their applications in multi-attribute decision making problems," Mathematics, vol. 7, no. 5, p. 413, 2019.

[42] M. Rafiq, S. Ashraf, S. Abdullah, T. Mahmood, and S. Muhammad, "The cosine similarity measures of spherical fuzzy sets and their applications in decision making," Journal of Intelligent \& Fuzzy Systems, vol. 36, no. 6, pp. 6059-6073, 2019.

[43] S. Ashraf and S. Abdullah, "Spherical aggregation operators and their application in multiattribute group decision-making," International Journal of Intelligent Systems, vol. 34, no. 3, pp. 493-523, 2019.

[44] V. Torra and Y. Narukawa, "On hesitant fuzzy sets and decision," in Proceedings of the IEEE International Conference on Fuzzy Systems, pp. 1378-1382, Jeju Island, South Korea, August 2009.

[45] V. Torra, "Hesitant fuzzy sets," International Journal of Intelligent Systems, vol. 25, no. 6, pp. a-n, 2010.

[46] R. M. Rodriguez, L. Martinez, and F. Herrera, "Hesitant fuzzy linguistic term sets for decision making," IEEE Transactions on Fuzzy Systems, vol. 20, no. 1, pp. 109-119, 2012.

[47] R. Li, J. Dong, and D. Wang, "Competition ability evaluation of power generation enterprises using a hybrid MCDM method under fuzzy and hesitant linguistic environment," Journal of Renewable and Sustainable Energy, vol. 10, no. 5, p. 055905, 2018.

[48] B. Oztaysi, S. C. Onar, E. Bolturk, and C. Kahraman, "Hesitant fuzzy analytic hierarchy process," in Proceedings of the 2015 IEEE International Conference on Fuzzy Systems (FUZZIEEE), Istanbul, Turkey, August 2015.

[49] F. Tüysüz and B. Şimşek, "A hesitant fuzzy linguistic term sets-based AHP approach for analyzing the performance evaluation factors: an application to cargo sector," Complex \& Intelligent Systems, vol. 3, no. 3, pp. 167-175, 2017.

[50] A. Camci, G. T. Temur, and A. Beskese, "CNC router selection for SMEs in woodwork manufacturing using hesitant fuzzy AHP method," Journal of Enterprise Information Management, vol. 31, no. 4, pp. 529-549, 2018.

[51] C. Acar, A. Beskese, and G. T. Temur, "Sustainability analysis of different hydrogen production options using hesitant fuzzy AHP," International Journal of Hydrogen Energy, vol. 43, no. 39, pp. 18059-18076, 2018.

[52] M. B. Ayhan, "An integrated hesitant fuzzy AHP and TOPSIS approach for selecting summer sport school," Sakarya University Journal of Science, vol. 22, no. 2, 2018.

[53] F. Samanlioglu and Z. Ayağ, "An intelligent approach for the evaluation of innovation projects," Journal of Intelligent \& Fuzzy Systems, vol. 38, no. 1, pp. 905-915, 2020.

[54] L. Anojkumar, M. Ilangkumaran, and V. Sasirekha, "Comparative analysis of MCDM methods for pipe material selection in sugar industry," Expert Systems with Applications, vol. 41, no. 6, pp. 2964-2980, 2014.
[55] Z. Wu, J. Ahmad, and J. Xu, "A group decision making framework based on fuzzy VIKOR approach for machine tool selection with linguistic information," Applied Soft Computing, vol. 42, pp. 314-324, 2016.

[56] D. Yong, "Plant location selection based on fuzzy TOPSIS," The International Journal of Advanced Manufacturing Technology, vol. 28, no. 7-8, pp. 839-844, 2005.

[57] D. Yu, "Triangular hesitant fuzzy set and its application to teaching quality evaluation," Journal of Information and Computational Science, vol. 10, no. 7, pp. 1925-1934, 2013.

[58] A. Başar, "Hesitant fuzzy pairwise comparison for software cost estimation: a case study in Turkey," Turkish Journal of Electrical Engineering \& Computer Sciences, vol. 25, no. 4, pp. 2897-2909, 2017.

[59] H. Liu and R. M. Rodríguez, "A fuzzy envelope for hesitant fuzzy linguistic term set and its application to multicriteria decision making," Information Sciences, vol. 258, pp. 220-238, 2014.

[60] D. Filev and R. R. Yager, "On the issue of obtaining OWA operator weights," Fuzzy Sets and Systems, vol. 94, no. 2, pp. 157-169, 1998.

[61] Y.-M. Wang and Y. Luo, "On rank reversal in decision analysis," Mathematical and Computer Modelling, vol. 49, no. 5-6, pp. 1221-1229, 2009.

[62] V. Belton and T. Gear, "On a short-coming of Saaty's method of analytic hierarchies," Omega, vol. 11, no. 3, pp. 228-230, 1983.

[63] S. Schenkerman, "Avoiding rank reversal in AHP decisionsupport models," European Journal of Operational Research, vol. 74, no. 3, pp. 407-419, 1994.

[64] H. Alharthi, N. Sultana, A. Al-Amoudi, and A. Basudan, “An analytic hierarchy process-based method to rank the critical success factors of implementing a pharmacy barcode system," Perspectives in Health Information Management, vol. 12, 2015.

[65] T. L. Saaty, "Group decision making and the AHP," in The Analytic Hierarchy Process, Springer, Berlin, Germany, 1989. 